\title{
The safety and efficacy of high-dose daptomycin combined with rifampicin for the treatment of Gram-positive osteoarticular infections
}

\author{
Kheeldass Jugun • Pierre Vaudaux • Jorge Garbino • \\ Leonardo Pagani • Pierre Hoffmeyer • Daniel Lew • \\ Ilker Uçkay
}

Received: 7 February 2013 / Accepted: 26 February 2013 /Published online: 22 March 2013

(C) Springer-Verlag Berlin Heidelberg 2013

\begin{abstract}
Purpose Treatment of Gram-positive osteoarticular infections requires an adequate surgical approach combined with intensive antimicrobial therapy. The aim of this study was to evaluate the safety and efficacy of a combined regimen of high-dose daptomycin and rifampicin, in patients with various types of Gram-positive osteoarticular infections.

Methods This single centre, non-comparative, prospective study evaluated the safety and efficacy of a combined regimen of intravenous daptomycin $(8 \mathrm{mg} / \mathrm{kg} / \mathrm{day})$ and oral rifampicin $(600 \mathrm{mg} /$ day $)$ in patients with Gram-positive osteoarticular infections, with a minimal follow-up of one year. Creatine phosphokinase, transaminases, bilirubinaemia, and serum creatinine, were measured at baseline and regular intervals.

Results The median daily doses of daptomycin and rifampicin, administered for a median duration of 21 (range, 10-122) days to 16 patients (median age, 63.5 years; 11 males, five females) presenting with staphylococcal $(n=15)$ or streptococcal $(n=1)$ osteoarticular infections, were 8.15 (range, 6.6-8.9) $\mathrm{mg} / \mathrm{kg} /$ day and 600 (range, 600-900) $\mathrm{mg} /$ day, respectively. The combined regimen of daptomycin and rifampicin was well tolerated by all except one patient, without requiring
\end{abstract}

K. Jugun · P. Hoffmeyer · I. Uçkay

Orthopaedic Surgery Service, Geneva University Hospital and

Medical School, Geneva, Switzerland

P. Vaudaux $(\bowtie) \cdot J$. Garbino $\cdot$ L. Pagani $\cdot$ D. Lew $\cdot$ I. Uçkay Service of Infectious Diseases, Geneva University Hospital and Medical School, 4 Rue Gabrielle Perret-Gentil,

1211, Geneva 14, Switzerland

e-mail: Pierrevaudaux@yahoo.fr

P. Vaudaux

e-mail: Pierre.vaudaux@unige.ch treatment adjustment or discontinuation. One patient developed allergic responses probably due to rifampicin after 42 days. Fifteen (94\%) patients showed favourable clinical and microbiological outcomes.

Conclusions The combined regimen of high-dose daptomycin and rifampicin was well tolerated and may provide a useful alternative to standard glycopeptide therapy for Gram-positive osteoarticular infections.

\section{Introduction}

Treatment of osteoarticular infections due to coagulasenegative staphylocococci (CNS), Staphylococcus aureus, or Streptococcus sp, in particular those associated with artificial devices used for osteosynthesis or permanent prosthetic implants [1], requires an adequate surgical approach combined with long-term antimicrobial therapy. Very few antimicrobial agents remain available for therapy of osteoarticular infections that are due to multi-resistant Gram-positive pathogens, in particular methicillin-resistant S. aureus (MRSA). Besides glycopeptides that remain the mainstay for therapy of MRSA deep-seated infections, some recently developed agents that are active against both susceptible and multi-resistant, gram-positive pathogens including MRSA, in particular daptomycin [2-6] and linezolid [7, 8], have been recently evaluated for therapy of osteoarticular infections.

Daptomycin is a lipopeptide antibiotic that has a rapid bactericidal activity in vitro and in vivo, but this bactericidal activity is concentration-dependent and its optimal expression may require plasma levels equivalent to eight times the lipopeptide minimum inhibitory concentration (MIC) for 
some strains $[9,10]$. The relatively high protein binding and low volume of distribution of daptomycin recorded in healthy volunteers [11] represent a difficult challenge for defining a dosing schedule exerting optimal activity against various categories of deep-seated bacterial infections.

A once-daily dosing regimen of $4 \mathrm{mg} / \mathrm{kg} /$ day daptomycin was initially approved for the treatment of complicated skin and skin-structure infections due to gram-positive bacteria [12]. Subsequently, daptomycin was found effective for therapy of $S$. aureus bacteremia and right-sided endocarditis at a once-daily dosing regimen of $6 \mathrm{mg} / \mathrm{kg} /$ day [13]. While early clinical studies with twice-daily regimens of daptomycin raised concerns about potential myopathy characterised by high elevations ( $>10$-fold) in serum creatine phosphokinase $[9,14]$, further pharmacokinetic studies demonstrated the safety of higher doses of daptomycin, such as $8 \mathrm{mg} / \mathrm{kg} / \mathrm{day}$, when they were administered once daily for 14 days in healthy volunteers [11].

We performed a single centre, non-comparative, prospective study to evaluate the safety and efficacy of a combined regimen of high-dose daptomycin $(8 \mathrm{mg} / \mathrm{kg} /$ day $)$ and rifampicin $(600 \mathrm{mg} /$ day $)$, for treatment of patients with Grampositive osteoarticular infections.

\section{Materials and methods}

\section{Patient eligibility}

The design of our single-centre, prospective, noncomparative study, which required the informed consent of all patients included, was approved by the Ethics Committee of our institution and Swissmedic (No 08-061; NAC 08-001). Patients aged 18 years or older, presenting with osteoarticular infections at the Orthopaedic Clinic of the Geneva University Hospital, were eligible for inclusion. Pregnant or lactating women were excluded. Appropriate diagnoses included bone or/and joint infection that was associated or not with osteosynthesis devices or orthopaedic prostheses, involving the presence of the same microbial pathogen in several intraoperative or purulent, deep specimens.

Patients were excluded if they had received other antimicrobial agents for more than 72 hours prior to the combined regimen of daptomycin and rifampicin. Other exclusion criteria involved documented allergic reactions to daptomycin or rifampicin, or infections with microbial pathogens resistant to any of those antibiotics. Patients with severe renal insufficiency (creatinine clearance under $30 \mathrm{~mL} / \mathrm{min}$ ), baseline creatine phosphokinase $(\mathrm{CPK})$ values that were more than five times greater than upper normal (188 Units/L) limits, or severe hepathopathy (CHILD C with transaminases and/or biluribinemia over five times greater than upper normal limits) were also excluded.
Due to the potentially adverse effects of the study drugs, including hepatitis, all patients included were advised to refrain from alcohol consumption during the study period.

Other exclusion criteria were epilepsy or receipt of major immunosuppressive therapy (cyclosporine, rapamune, tacrolimus, prednisone dosage over $20 \mathrm{mg} /$ day).

Study design and treatment

All study patients with Gram-positive osteoarticular infections received once-daily regimens of intravenous daptomycin ( $8 \mathrm{mg} / \mathrm{kg}$ by $30-\mathrm{min}$ infusion) combined with oral rifampicin ( $600 \mathrm{mg} /$ day). Treatment duration was determined by the medical team and guided by the clinical evolution and surgical treatment of each patient.

\section{Clinical and laboratory parameters}

The clinical evolution was evaluated by the medical staff at baseline and weekly intervals throughout the whole duration of daptomycin-rifampicin therapy. Ambulatory patients were evaluated at the Policlinic of Surgery according to clinical needs.

Clinical laboratory parameters were also measured at baseline and checked weekly during daptomycin-rifampicin therapy, including clinical chemistry and other blood parameters, CPK, serum creatinine levels, aspartate aminotransferase (ASAT) and alanine transaminase (ALAT), bilirubinemia. Microbiological cultures and antibiotic susceptibility testing were processed under standard conditions.

Follow-up evaluation was performed six to eight weeks after the end of daptomycin-rifampin therapy. Final followup assessments were undertaken at least one year after the end of antibiotic therapy.

\section{Safety evaluation}

Patients were frequently monitored for the incidence of clinically significant adverse events during hospitalisation and at intervals during follow-up out patient visits. The incidence of adverse effects was also checked by laboratory detection of significant elevations in enzymatic markers for hepatic (upper normal limits: ASAT 42 units/L, ALAT 42 units/L), kidney (serum creatinine), or muscle (CPK) toxicity (upper normal limit: $188 \mathrm{IU} / \mathrm{L}$ ).

Discontinuation of the daptomycin-rifampicin protocol was required in the event of of any major clinical side effect (hepatitis with greater than five times elevation in transaminases levels, renal insufficiency with a more than two-fold increase in serum creatinine, CPK elevations $>$ $500 \mathrm{IU} / \mathrm{L}$ ), or in case of a treatment-emergent resistance or microbiologically documented superinfection by another bacterial species during therapy. 
Clinical response

The primary objective was to evaluate the safety profile of the daptomycin-rifampicin combination. A secondary objective was the clinical efficacy of the daptomycin-rifampicin combined therapy. The clinical efficacy was considered successful if no major surgical procedure or no major change in antibiotic therapy was required during the therapeutic protocol, and if clinical resolution and microbiological eradication of the pathogen were assessed after at least one-year follow-up period. Treatment failure was defined as inadequate clinical response or microbiologically documented relapse of the osteoarticular infection, occurring during the daptomycin-rifampicin protocol or its follow-up period.

\section{Statistical analyses}

Categorical variables were expressed as percentages and numerical data as median and range.

\section{Results}

Sixteen patients with osteoarticular infections were included. Their detailed demographic, clinical, and laboratory data are presented in Table 1. The median age was 63.5 (range, 26-85) years. Five $(31 \%)$ patients were females and 11 (69 \%) were males. Thirteen $(81 \%)$ patients had implanted devices, of which seven were for osteosynthesis and six for arthroplasty. One patient had a vascular prosthesis.

Fifteen (94\%) patients had staphylococcal and one patient had a streptococcal (Streptoccocus mitis) infections. Three $(17 \%)$ patients were infected with methicillin-resistant Staphylococcus epidermidis (MRSE), five (31\%) with methicillinsusceptible $S$. aureus (MSSA), and seven (44\%) with MRSA.

Underlying conditions included diabetes for five (31\%) patients, low-level immunosuppressive therapy for two patients, namely, one with kidney graft and one with autoimmune disease, and statin therapy for two diabetic patients with peripheral arterio-occlusive disease. One patient had hepatitis $\mathrm{C}$.

The median duration of hospitalisation was 21.5 (range, six to 126) days. The median number of surgical procedures was two (range, one to six). While five patients (31\%) had a single surgical procedure, 11 patients $(69 \%)$ had two or more procedures.

Daptomycin was i.v. administered once-daily at a median dose of 8.15 (range, 6.6-8.9) $\mathrm{mg} / \mathrm{kg} /$ day. Rifampicin was orally administered once-daily at a median dose of 600 (range, 600-900) mg/day, to all patients except one who received $900 \mathrm{mg} /$ day (Table 1). Eleven patients received the daptomycin-rifampicin regimen as first-line therapy and five patients as second-line therapy.

The combined regimen of high-dose daptomycin and rifampicin was administered for a median duration of 21 (range, ten to 122) days and was well supported by all patients, including two who received concomitant statin therapy. Only minor side effects (mild fatigue or mild myalgia) occurred in two patients. This good tolerance was assessed by laboratory testing of enzymatic toxicity markers, which showed no clinically significant elevation throughout antibiotic therapy for any of the patients. Maximal values of repeatedly assayed CPK ranged from five to $305 \mathrm{IU} / \mathrm{L}$ (median, $88 \mathrm{IU} / \mathrm{L}$ ) (Table 1). Maximal values of transaminases ranged from 15 to 52 IU/L (median, $20.5 \mathrm{IU} / \mathrm{L})$. Moreover, serum creatinine levels did not change significantly during therapy.

While no significant, clinically or laboratory-documented adverse effect occurred that required adjustment or discontinuation of daptomycin therapy, one patient developed an ileus and a rash clinically attributed to rifampicin after 42 days of combined antibiotic therapy, which led to discontinuation of rifampicin administration.

Evaluation of treatment outcomes was performed over a median follow-up period of 473 (range, 373-900) days. This global evaluation represented the sum of all medical and surgical procedures, including additional antimicrobial therapy that was administered to 11 patients after the combined regimen of rifampicin and daptomycin. Six of the seven patients with osteosynthesis devices, and five of six patients with arthroplasty prostheses, received additional, orally-administered antibiotic regimens after leaving the hospital for median periods of 25 (range, 16-344) and 170 (range, 111-658) days, respectively.

A single patient who was diagnosed with bacteraemia combined with a para-valvular abscess during daptomycinrifampicin therapy and who died from endocarditis and sepsis on day 15 was not included in outcome evaluation. All other 15 patients had an infection-free follow-up, including two patients who had bacteraemia during therapy, involving the same MSSA osteoarticular pathogen but without endocardial involvement, and another patient who had a vascular prosthesis that led to limb amputation. No emergence of daptomycin or rifampicin-resistant isolate occurred in any patient, which would have required their exclusion from the therapeutic protocol.

Orthopaedic implants were removed in eight patients. Prostheses were retained in three patients, permanently removed in one patient, or totally or partially exchanged in two patients. Osteosynthesis devices were retained in one patient, permanently removed in four patients, and exchanged in two patients.

\section{Discussion}

Recent studies indicate a growing interest in the design of novel therapeutic protocols for deep-seated multi-resistant 


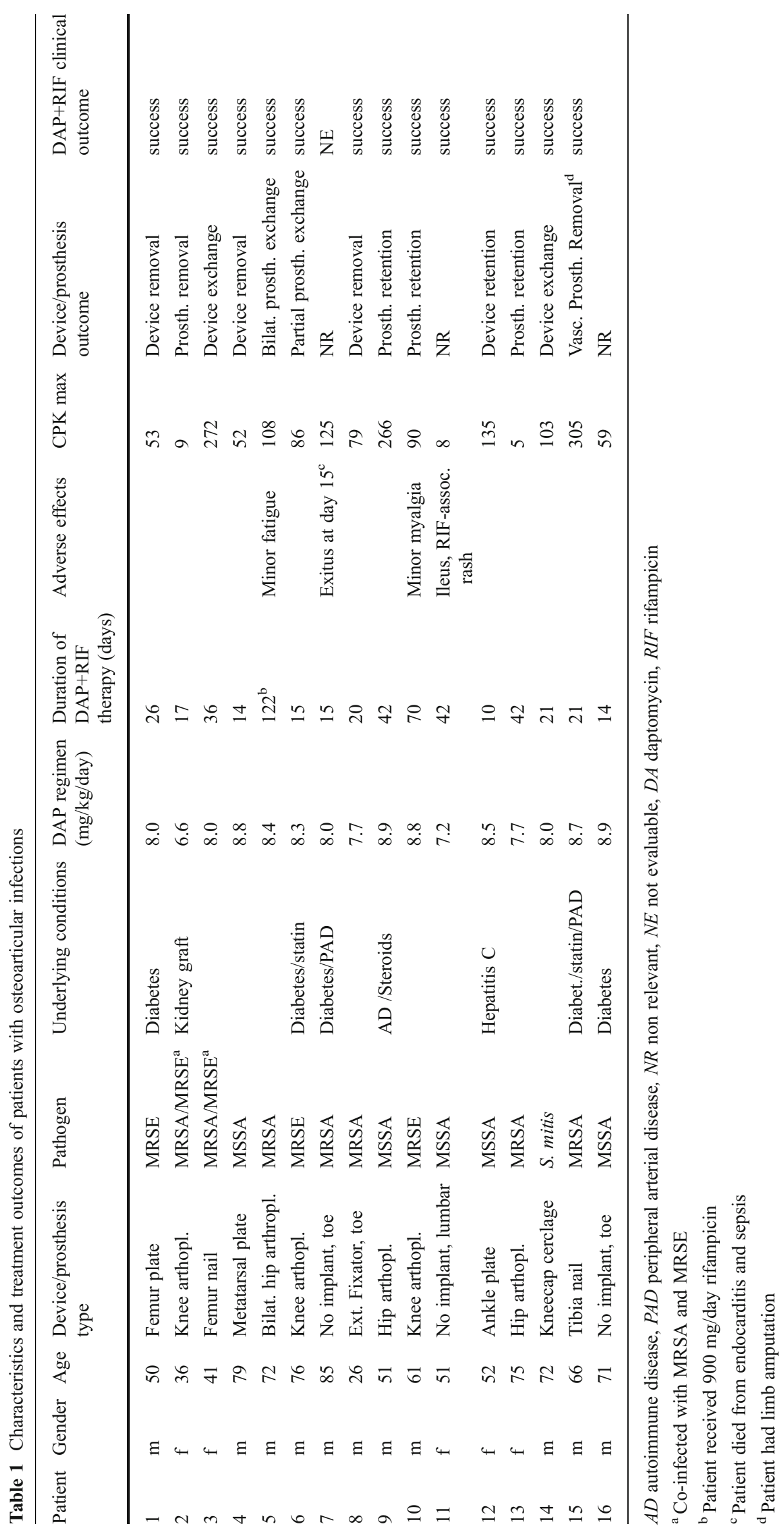


Gram-positive infections, as alternatives to the standard glycopeptide therapy [2-8]. Daptomycin has attractive properties for treating osteoarticular infections, because of its rapid bactericidal activity against susceptible as well as multi-resistant, Gram-positive isolates. Emergence of daptomycin resistance remains a rare event in staphylococci and is limited to individual case reports $[9,10]$. While recent clinical data assessed the efficacy and safety of high-dose daptomycin in Gram-positive osteoarticular infections [2-6], no specific study evaluated the combined regimen of high-dose daptomycin $(8 \mathrm{mg} / \mathrm{kg} /$ day $)$ and rifampicin $(600 \mathrm{mg} /$ day $)$ in this context. In view of the quite variable (from 300 to $1,200 \mathrm{mg} /$ day) regimens of rifampicin that have been described in the literature, official recommendations for a standard rifampicin regimen in the context of osteoarticular infections would be most appropriate. Nevertheless, the median daily dose of $600 \mathrm{mg}$ /day of rifampicin is congruent with Swiss and US expert recommendations and warrant continuously active levels of this antimicrobial agent throughout therapy.

The safety of high dose (over $6 \mathrm{mg} / \mathrm{kg} /$ day) daptomycin, concomitant or not with statin therapy, was evaluated by Parra-Ruiz et al. in 43 and 25 patients with prosthetic joint and diabetic infections, respectively [4]. While resolution of infection was documented in 33/43 (77\%) patients with prosthetic joint infections and 17/25 (68\%) patients with diabetic foot infections, the proportion of staphylococcal infections in those two groups was not indicated. Interestingly, the overall rate of biochemically detected muscular toxicity in all daptomycin-treated patients $(n=104)$, defined as CPK levels $>1,000 \mathrm{UI} / \mathrm{L}$, was approximately $10 \%$. In contrast, no clinically significant toxicity was observed and daptomycin therapy was completed in all cases. This study also reported that concomitant administration of daptomycin and statins was safe and not associated with an increased risk of rhabdomyolysis or myositis. In another retrospective study, Corona Perez-Cardona et al. described 20 patients with knee or hip periprosthetic joint infections, who received an average daptomycin dose of $6.6 \mathrm{mg} / \mathrm{kg} /$ day for a mean duration of 44.9 days [3]. Only 14 of those patients were evaluable for outcome, yielding an overall success rate of $78.6 \%$. Since only four patients were infected with MSSA or MRSA in that study, the significance of daptomycin efficacy data against $S$. aureus prosthetic joint infections is limited. Noteworthy, severe side effects occurred in two patients, namely, one case of acute renal failure due to massive rhabdomyolysis and one of eosinophilic pneumonia, which resolved with appropriate therapy after the end of daptomycin administration [3].

The safety and efficacy of high dose daptomycin for treating osteoarticular infections was confirmed by two recent studies, the first one targeting patients with prosthetic devices undergoing two-stage revision arthroplasty [6], and the second of non-hardware associated osteomyelitis patients [5]. In both studies, clinically and laboratory documented daptomycinrelated adverse effects ranged from five to over $20 \%$.

It is remarkable that our prospective, observational study did not reveal any daptomycin-related, clinically significant side effects, in patients with osteoarticular infections treated with combined regimens of high-dose daptomycin and rifampicin for several weeks. Tolerability of the daptomycinrifampicin regimen was confirmed by laboratory assessment of enzymatic markers for muscular, liver or kidney toxicity, which showed no significant elevations, except for one patient who developed allergic responses probably due to rifampicin. A significant proportion of those patients had underlying diseases, such as diabetes, immunocompromised conditions or vascularisation problems and were facing multiple surgical procedures. Finally, additional risk factors for antibiotic treatment failure were the presence of implanted devices or prostheses in most patients, combined with the presence of multiresistant strains of $S$. aureus or $S$. epidemidis.

While recent clinical studies afford no evidence for a dosedependent daptomycin-related muscular toxicity, there is a growing consensus for an improved therapeutic efficacy of daptomycin against deep-seated Gram-positive pathogens, when this antibiotic is used at high-dose regimens (over $6 \mathrm{mg} / \mathrm{kg} /$ day). In particular, high-dose daptomycin is assumed to improve daptomycin's bone penetration [3, 4]. The dose-related efficacy of daptomycin was also observed in animal models of foreign body infections [15-17]. Taken together, the safety and efficacy profiles of the combined regimen of high-dose daptomycin and rifampicin provide a useful alternative to standard glycopeptide therapy for osteoarticular infections due to susceptible as well as multiresistant Gram-positive pathogens. Further studies evaluating a larger group of patients with Gram-positive orthopaedic infections are required to evaluate the rate of clinically significant side effects and the potential occurrence of therapy failures or/and emergence of daptomycin resistance.

Acknowledgments This study was supported in part by an educational research grant from Novartis AG and research grant 3200B0-108401 (D.L) from the Swiss National Science Foundation. These funding organizations exerted no influence on the content of this manuscript. We thank all physicians and nurses involved in patients' care.

\section{References}

1. Zimmerli W, Trampuz A, Ochsner PE (2004) Prosthetic-joint infections. N Engl J Med 351:1645-1654

2. Falagas ME, Giannopoulou KP, Ntziora F, Papagelopoulos PJ (2007) Daptomycin for treatment of patients with bone and joint infections: a systematic review of the clinical evidence. Int $\mathrm{J}$ Antimicrob Agents 30:202-209

3. Corona Pérez-Cardona PS, Barro Ojeda V, Rodriguez Pardo D, Pigrau Serrallach C, Guerra Farfán E, Amat Mateu C, Flores 
Sanchez X (2012) Clinical experience with daptomycin for the treatment of patients with knee and hip periprosthetic joint infections. J Antimicrob Chemother 67:1749-1754

4. Parra-Ruiz J, Dueñas-Gutiérrez C, Tomás-Jiménez C, LinaresPalomino JP, Garrido-Gomez J, Hernández-Quero J (2012) Safety analysis of high dose ( $>6 \mathrm{mg} / \mathrm{kg} /$ day $)$ daptomycin in patients with concomitant statin therapy. Eur J Clin Microbiol Infect Dis $31: 1771-1774$

5. Gallagher JC, Huntington JA, Culshaw D, McConnell SA, Yoon M, Berbari E (2012) Daptomycin therapy for osteomyelitis: a retrospective study. BMC Infect Dis 12:133

6. Byren I, Rege S, Campanaro E, Yankelev S, Anastasiou D, Kuropatkin G, Evans R (2012) Randomized controlled trial of the safety and efficacy of daptomycin versus standard-of-care therapy for management of patients with osteomyelitis associated with prosthetic devices undergoing two-stage revision arthroplasty. Antimicrob Agents Chemother 56:5626-5632

7. Nguyen S, Pasquet A, Legout L, Beltrand E, Dubreuil L, Migaud H, Yazdanpanah Y, Senneville E (2009) Efficacy and tolerance of rifampicin-linezolid compared with rifampicin-cotrimoxazole combinations in prolonged oral therapy for bone and joint infections. Clin Microbiol Infect 15:1163-1169

8. Shinjoh M, Iketani O, Watanabe K, Shimojima N, Kudo M, Yamagishi H, Shimada H, Sugita K, Takahashi T, Mori T, Hasegawa N, Iwata S (2012) Safety and efficacy of linezolid in 16 infants and children in Japan. J Infect Chemother 18:591-596

9. Tally FP, Zeckel M, Wasilewski MM, Carini C, Berman CL, Drusano GL, Oleson FB Jr (1999) Daptomycin: a novel agent for gram-positive infections. Expert Opin Investig Drugs 8:1223-1238

10. Kern WV (2006) Daptomycin: first in a new class of antibiotics for complicated skin and soft-tissue infections. Int $\mathrm{J}$ Clin Pract 60:370-378
11. Dvorchik BH, Brazier D, DeBruin MF, Arbeit RD (2003) Daptomycin pharmacokinetics and safety following administration of escalating doses once daily to healthy subjects. Antimicrob Agents Chemother 47:1318-1323

12. Arbeit RD, Maki D, Tally FP, Campanaro E, Eisenstein BI (2004) The safety and efficacy of daptomycin for the treatment of complicated skin and skin-structure infections. Clin Infect Dis 38:1673-1681

13. Fowler VG Jr, Boucher HW, Corey GR, Abrutyn E, Karchmer AW, Rupp ME, Levine DP, Chambers HF, Tally FP, Vigliani GA, Cabell CH, Link AS, DeMeyer I, Filler SG, Zervos M, Cook P, Parsonnet J, Bernstein JM, Price CS, Forrest GN, Fätkenheuer G, Gareca M, Rehm SJ, Brodt HR, Tice A, Cosgrove SE (2006) Daptomycin versus standard therapy for bacteremia and endocarditis caused by Staphylococcus aureus. N Engl J Med 355:653665

14. Oleson FB Jr, Berman CL, Kirkpatrick JB, Regan KS, Lai JJ, Tally FP (2000) Once-daily dosing in dogs optimizes daptomycin safety. Antimicrob Agents Chemother 44:2948-2953

15. John A-K, Baldoni D, Haschke M, Rentsch K, Schaerli P, Zimmerli W, Trampuz A (2009) Efficacy of daptomycin in implant-associated infection due to methicillin-resistant Staphylococcus aureus: importance of combination with rifampin. Antimicrob Agents Chemother 53:2719-2724

16. Vaudaux P, Francois P, Bisognano C, Li D, Lew DP, Schrenzel J (2003) Comparative efficacy of daptomycin and vancomycin in the therapy of experimental foreign body infection due to Staphylococcus aureus. J Antimicrob Chemother 52:89-95

17. Schaad HJ, Bento M, Lew DP, Vaudaux P (2006) Evaluation of high-dose daptomycin for therapy of experimental Staphylococcus aureus foreign body infection. BMC Infect Dis 6:74 